\title{
USO DE POLEIROS ARTIFICIAIS NA RECOMPOSIÇÃO DO BANCO DE SEMENTES EM ÁREA DE MATA CILIAR
}

\section{USE OF ARTIFICIAL PERCHES IN THE RESTORATION OF THE SEED BANK IN AN AREA OF RIPARIAN FOREST}

\author{
Jussara Gislaine Servilheri (D), Fernando Henrique de Oliveira (D), Wellington Fernando Lemes (D), \\ José Felinto Barbosa* (iD
}

UNINGÁ - Centro Universitário Ingá, Maringá, PR, Brasil.

*j_felinto@yahoo.com.br

\section{RESUMO}

Matas ciliares são consideradas áreas de preservação permanente e incluem todos os tipos de vegetação que se vinculam às margens de rios com grande importância para a manutenção dos recursos hídricos. A recuperação natural de uma área degradada depende da chegada de sementes a este local, que podem ser transportadas por diversos meios, entre os quais, por aves. O objetivo do presente estudo foi avaliar o papel de poleiros artificiais como atrativos para aves e seu efeito na recomposição do banco de sementes de uma área de mata ciliar degradada, localizada no município de Doutor Camargo / Paraná - Brasil. Foram instalados 16 poleiros artificiais com coletores para as fezes das aves e 16 coletores sem a influência dos poleiros (controle). Os dados foram obtidos entre junho e setembro de 2010. Foram amostradas 3.673 sementes, das quais 2.945 estavam em meio às fezes de aves. Foram constatadas diferenças significativas na quantidade de sementes amostradas em coletores sem poleiros e com poleiros artificiais, demonstrando a importância dos poleiros como ferramenta auxiliar no processo de recuperação de áreas degradadas.

Palavras-chave: Aves frugívoras. Ornitocoria. Recuperação ambiental.

\begin{abstract}
Riparian forests are considered permanent preservation areas and include all types of vegetation that are linked to the banks of rivers with great importance for the maintenance of water resources. The natural regeneration of a degraded area depends on the arrival of seeds to this place, which can be transported by various means, including birds. The objective of the present study was to evaluate the role of artificial perches as attractive to birds and their effect on the restoration of the seed bank of an area of degraded riparian forest, located in the city of Doutor Camargo / Paraná - Brazil. 16 artificial perches were installed with collectors for bird droppings and 16 collectors without the influence of perches (control). The data were obtained between June and September 2010. 3,673 seeds were sampled, of which 2,945 were in the middle of bird droppings. Significant differences were found in the amount of seeds sampled in collectors without perches and with artificial perches, demonstrating the importance of perches as an auxiliary tool in the process of restoring degraded areas.
\end{abstract}

Keywords: Environmental restoration. Frugivorous birds. Ornithocory. 


\section{INTRODUÇÃO}

As matas ciliares incluem todos os tipos de formações vegetais das margens dos rios, córregos, lagos, represas e nascentes e são consideradas como áreas de preservação permanente (BRASIL, 2012). A relação dessas matas com o ambiente hídrico que margeiam é de extrema importância, pois funcionam como verdadeiros cílios protetores, sem os quais os corpos de água sofrem degradação. De acordo com Kuntschik, Eduarte e Uehara (2014), as matas ciliares apresentam numerosas funções, entre as quais: promovem efeito de filtro e tampão, uma vez que colaboram com a qualidade e a quantidade de água ao reterem excesso de sedimentos que chegam aos corpos de água; evitam deslizamentos de terra e assoreamento dos rios; protegem as ribanceiras, pela rede formada pelas raízes; formam corredores ecológicos, locais de altíssima diversidade biológica e fixação do gás carbônico.

Apesar de sua grande importância, a situação atual de grande parte das matas ciliares é preocupante. Muitos cursos d'água apresentam formações ripárias com dimensões muito abaixo das determinadas pela legislação, ou ainda não apresentam nenhuma formação. As matas ciliares são ecossistemas utilizados pelo homem, devido, principalmente, aos seus solos férteis e úmidos, que são substituídos por áreas de produção agrícola e pecuária, como observado por Vibrans et al. (2011) e Sevegnani et al. (2012).

Devido à conscientização ecológica e à legislação ambiental, muitas iniciativas de restauração dessas matas têm sido observadas no Brasil (CHABARIBERY et al., 2008; LACERDA; FIGUEIREDO, 2009; BRAGA; MENDES; FONSECA, 2010). Tanto técnicas de regeneração artificial quanto natural são utilizadas. De acordo com Barnett e Baker (1991), a regeneração artificial apresenta maior facilidade de manejo e a possibilidade de regeneração de áreas extremamente degradadas, onde já não existe a produção de sementes. As técnicas mais comuns são o plantio de mudas, ou silvicultura, e a semeadura direta. Alvarenga (2004) destaca o plantio de mudas de espécies dos diferentes grupos sucessionais como a prática mais utilizada, mas a escolha depende da condição do terreno, do valor a ser investido e dos objetivos da recuperação.

A regeneração natural, por sua vez, é importante em áreas que sofreram grandes alterações e permite que as espécies se adaptem melhor a cada nova fase da sucessão ecológica. Em muitos casos o cercamento da área tem sido a única técnica utilizada, parando a degradação e permitindo a recomposição da área (CARPANEZZI, 2005), entretanto, áreas muito degradadas podem perder a capacidade de regeneração natural, principalmente devido à ausência de um banco de sementes no solo. $\mathrm{O}$ banco de sementes é formado pelas sementes vivas, produzidas em determinada área ou transportadas de outros locais, presentes no perfil do solo, incluindo aquelas superficiais (SAATKAMP; POSCHLOD; VENABLE, 2014). De acordo com Barbosa et al. (2009), a dispersão de sementes é muito importante no processo de regeneração natural de muitas espécies e na organização de novos ecossistemas, devido a sua influência sobre a composição, a estrutura e a dinâmica das populações de plantas que podem se estabelecer na área.

Levando em consideração os principais pontos da sucessão florestal, Reis et al. (2003) utilizaram a técnica de restauração ecológica na forma de nucleação em mosaicos, semelhantes aos encontrados no ambiente. Essa técnica gera um aumento da resiliência ambiental por se basear na ativação do potencial de autorregeneração da comunidade, fornecendo condições de abrigo, alimentação e reprodução, responsáveis por criar um novo nicho e acelerar o processo de sucessão, que se espalha por toda a área. Entre as técnicas de nucleação estão a transposição do solo, a transposição de galharia, os poleiros artificiais e a coleta de sementes com manutenção da variabilidade genética. A principal vantagem dessas técnicas, além do baixo custo, é o grande potencial de reprodução da composição e estrutura da vegetação local, dificilmente atingida por meio do plantio de mudas.

Dessa forma, diante da importância da restauração ambiental e da diversidade de técnicas utilizadas, o objetivo desse estudo foi avaliar a eficiência de poleiros artificiais na recomposição do banco de sementes em área de mata ciliar degradada. 


\section{MATERIAL E MÉTODOS}

O estudo foi conduzido no município de Doutor Camargo (Paraná), em uma propriedade rural às margens do Córrego Itatinga (S23 32' 29', W52 ${ }^{\circ} 13^{\prime} 37^{\prime}$ '), inserido na Bacia Hidrográfica do Rio Ivaí. O córrego tem uma largura média de $3 \mathrm{~m}$ e a mata ciliar na área da propriedade se encontra em processo de restauração em diferentes estágios de sucessão.

O desenho experimental foi estabelecido em duas linhas de poleiros artificiais, sendo uma instalada no interior da borda da mata em recuperação e a outra, na margem da borda, a uma distância de $5 \mathrm{~m}$ (Figura 1). Em cada linha foram intercalados coletores de sementes associados aos poleiros artificiais com coletores de sementes que não possuíam poleiros (Figura 1C). Estes coletores de sementes sem poleiros foram instalados para atuarem como controle. Deste modo, em cada uma dessas linhas havia 16 coletores de sementes, instalados a $2,5 \mathrm{~m}$ um do outro, e 8 poleiros artificiais. Estes poleiros artificiais foram feitos com estacas de bambu, fixas no solo e com 2,4 m de altura, cruzado, próximo ao ápice, por um bambu mais fino, de cerca de $90 \mathrm{~cm}$ de comprimento, para o pouso das aves. Os coletores de sementes foram elaborados com bambu e tecido de voal com as dimensões de $60 \mathrm{~cm}$ x $100 \mathrm{~cm}\left(0,6 \mathrm{~m}^{2}\right)$, instalados a $50 \mathrm{~cm}$ do solo, como sugerido por Reis et al. (2003). A soma da área dos coletores de sementes em cada tratamento foi de $4,8 \mathrm{~m}^{2}$.

Os dados foram coletados de junho a setembro de 2010. As fezes e sementes presentes nos coletores foram retiradas semanalmente e levadas ao laboratório para triagem com auxílio de estereomicroscópio. As sementes foram separadas em morfotipos, no Laboratório de Biologia do Centro Universitário Ingá - UNINGÁ e, posteriormente, identificadas com auxílio do Herbário da Universidade Estadual de Maringá (HUEM). As sementes com características de síndrome de dispersão autocórica e anemocórica, que não estavam em meio às fezes, e que, por acaso, foram observadas nos coletores, não foram consideradas. Para verificar os efeitos da presença de poleiros artificiais e da distância da borda da mata na chuva de sementes foram realizadas comparações entre médias com os testes Kruskal-Wallis e Tukey, com auxílio do programa Past $4.02^{\odot}$, elaborado por Hammer, Harper e Ryan (2001).

Figura 1 - Disposição dos poleiros artificiais e coletores de sementes na área de estudo
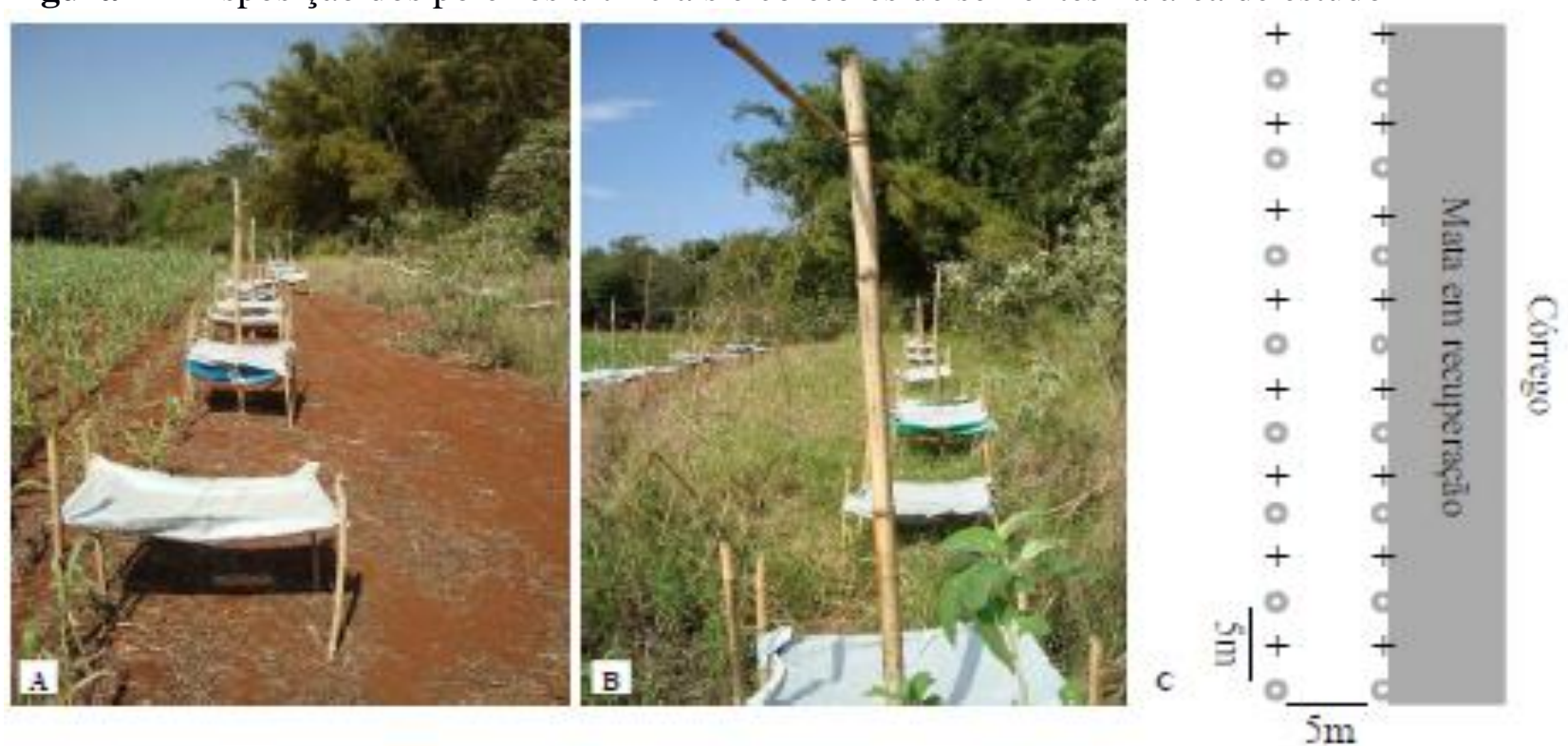

Notas: A: Poleiros artificiais e coletores de sementes instalados a $5 \mathrm{~m}$ da borda da mata em recuperação. B: Poleiros artificiais e coletores de sementes instalados na borda da mata em recuperação. C: Esquema da disposição dos poleiros e coletores de sementes na área de estudo: poleiros com coletores de sementes $(+)$ e coletores sem poleiros $(\mathbf{o})$.

Fonte: os autores. 


\section{RESULTADOS E DISCUSSÃO}

Foram amostradas 3.673 sementes nos coletores durante o período de estudo. Dessas, 2.945 estavam presentes nas fezes ou apresentavam resquícios de fezes de aves e foram consideradas em nossas análises. Os coletores localizados na linha de $5 \mathrm{~m}$ da borda da mata apresentaram maior número de sementes (1600) que aqueles localizados no interior da borda da mata (1345).

Os poleiros artificiais foram mais eficientes na linha de $5 \mathrm{~m}$ da borda da mata, onde foram registradas 1580 sementes nos coletores com poleiros artificiais, acarretando uma chuva de 329,17 sementes $/ \mathrm{m}^{2}$, enquanto nos coletores sem poleiros foram registradas somente 20 sementes, ou seja, 4,17 sementes $/ \mathrm{m}^{2}$. Nos coletores do interior da borda da mata, foi observada maior quantidade de sementes naqueles com poleiros (789), mas sem diferença expressiva para os coletores sem poleiros (556) (Tabela 1).

Os poleiros artificiais contribuíram significativamente para a recomposição do banco de sementes na área de mata degradada. A maior média semanal de sementes foi observada nos coletores instalados sob os poleiros artificiais a $5 \mathrm{~m}$ da borda da mata, com 131,67 $( \pm 119,25)$ sementes, sendo significativamente diferente dos coletores sem poleiros a $5 \mathrm{~m}$ da borda da mata, que apresentaram média de 1,67 \pm 4,66 sementes por semana [KW-H=22,28; $\mathrm{p}<0,001$; Tukey, $\alpha=0,05]$ (Figura 2). Nota-se um alto desvio padrão nas médias observadas, talvez pela variação na abundância de frutos durante as semanas avaliadas. Vale ressaltar que a eficiência dos poleiros artificiais como agentes facilitadores da dispersão de sementes já foi testada em diversos estudos (TOMAZI; ZIMMERMANN; LAPS, 2010; DIAS; UMETSU; BREIER, 2014; GUIDETTI et al., 2016) e os resultados favorecem a utilização dessa técnica como auxiliar em processos de recuperação de áreas degradadas.

Tabela 1 - Número de sementes amostradas nos coletores de sementes

\begin{tabular}{ccc}
\hline Tratamento & Total de sementes & Sementes $/ \mathbf{m}^{\mathbf{2}}$ \\
\hline CPB & 789 & 164,37 \\
SPB & 556 & 115,83 \\
CP5 & 1580 & 329,17 \\
SP5 & 20 & 4,17 \\
\hline
\end{tabular}

Notas: CPB: Coletores com poleiros na borda da mata. SPB: Coletores sem poleiros na borda da mata. CP5: Coletores com poleiros a $5 \mathrm{~m}$ da mata. SP5: Coletores sem poleiros a $5 \mathrm{~m}$ da mata.

Fonte: os autores.

Figura 2 - Número médio de sementes encontradas semanalmente nos coletores de sementes

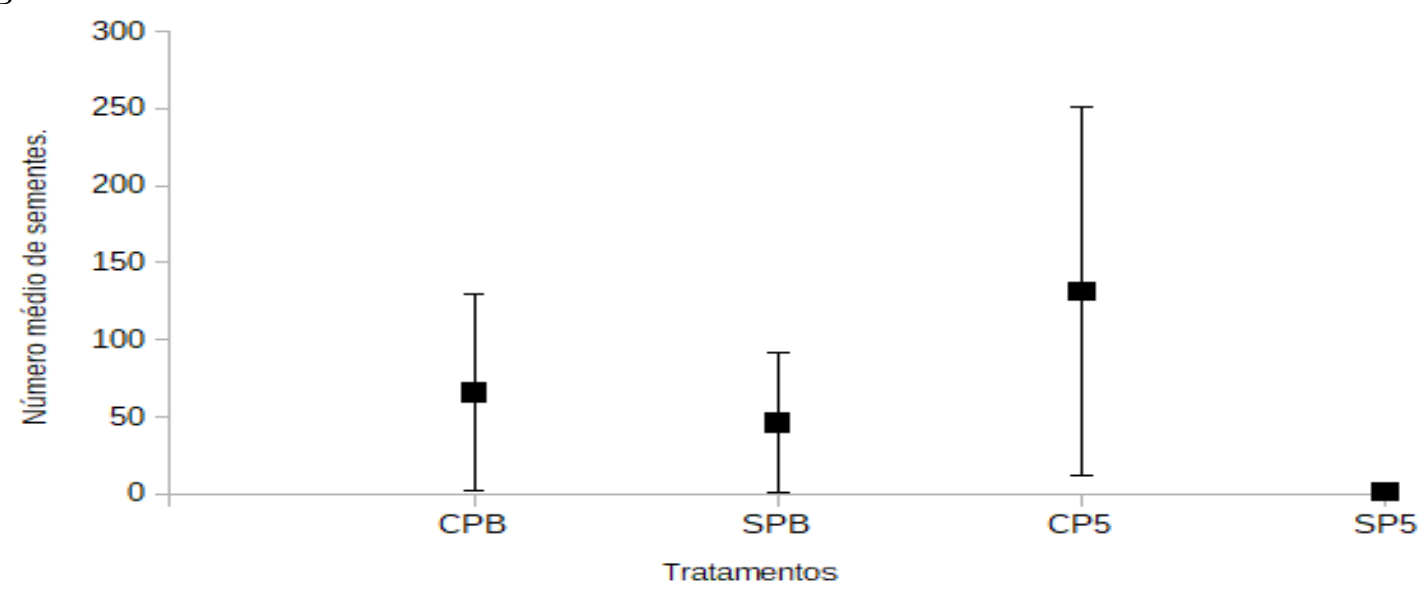

Notas: CPB: Coletores com poleiros na borda da mata. SPB: Coletores sem poleiros na borda da mata. CP5: Coletores com poleiros a $5 \mathrm{~m}$ da mata. SP5: Coletores sem poleiros a $5 \mathrm{~m}$ da mata.

Fonte: os autores. 
Os coletores associados ou não com poleiros artificiais na borda da mata apresentaram 65,75 $( \pm 63,39)$ e $46,33( \pm 44,92)$ sementes por semana, respectivamente. A quantidade expressiva de sementes em coletores sem poleiros artificiais pode ser justificada pela localização próxima à mata, onde as aves podem ter utilizado as próprias árvores e arbustos como poleiros naturais e suas fezes foram depositadas sobre esses coletores-controle. Ainda assim, é possível visualizar um incremento no número de sementes nos coletores associados aos poleiros, representando aquelas sementes oriundas das fezes de aves que utilizaram poleiros naturais mais o adicional de aves que utilizaram os poleiros artificiais. Bocchese et al. (2008) indicam a utilização de poleiros artificiais associados com poleiros naturais, como árvores remanescentes, para criar mais opções de poleiros e, assim, aumentar a diversidade de aves e sementes que chegam ao local.

Foram identificadas dez espécies diferentes de plantas distribuídas em seis famílias e mais duas espécies não identificadas (Tabela 2). Constata-se uma pequena diversidade de plantas que reflete a flora da região, em sua maior parte degradada ou em processo de recuperação.

Tabela 2 - Lista de espécies/morfoespécies de plantas amostradas nos coletores de sementes e identificadas a partir das sementes

\begin{tabular}{llcc}
\hline \multicolumn{1}{c}{ Família } & \multicolumn{1}{c}{ Espécie } & N & \% \\
\hline Anacardiaceae & Schinus terebinthifolia Raddi, 1820 & 18 & 0,61 \\
Apocynaceae & Tabernaemontana catharinensis A. DC., 1844 & 7 & 0,24 \\
Cecropiaceae & Cecropia pachystachya Trécul, 1847 & 1.102 & 37,42 \\
Myrtaceae & Psidium guajava L., 1753 & 98 & 3,33 \\
Poaceae & Megathyrsus maximus (Jacq.) B.K. Simon \& S.W.L. & 158 & 5,37 \\
& Jacobs, 2003 & 92 & 3,12 \\
& Sorghum sp. & 3 & 0,1 \\
& Triticum aestivum L., 1753 & 1.211 & 41,12 \\
Solanaceae & Solanum mauritianum Scop., 1788 & 82 & 2,78 \\
& Solanum sp1 & 83 & 2,82 \\
Indeterminada & Solanum sp2 & 89 & 3,02 \\
Indeterminada & Indeterminada 1 & 2 & 0,07 \\
\hline & Indeterminada 4 & 2945 & 100 \\
\hline
\end{tabular}

Fonte: os autores.

As espécies vegetais mais representadas nas amostragens foram Solanum mauritianum Scop., 1788 (Solanaceae) com 1211 sementes (41,12\%) e Cecropia pachystachya Trécul, 1847 (Cecropiaceae), com 1102 sementes (37,42\%). Solanum mauritianum é uma espécie nativa e está presente em muitas áreas antropizadas, borda de florestas e em florestas secundárias, com frutificação distribuída ao longo do ano (RUSCHEL; NODARI, 2011). Cecropia pachystachya também é uma espécie nativa, típica de formações secundárias e conhecida como embaúba (SOUZA; LORENZI, 2008), destacando-se que seus frutos são muito consumidos por pássaros (SANTOS; FRANCHIN; NUNES, 2017) e morcegos (SATO; PASSOS; NOGUEIRA, 2008). Assim, considerando-se a pequena diversidade de espécies vegetais na área de estudo, elas representaram importante recurso aos animais. Ao oferecermos atrativos para o pouso das aves, auxiliamos na recomposição do banco de sementes de áreas degradadas a partir de plantas presentes na própria região, visto que vários indivíduos dessas espécies ocorriam próximos à área de estudo.

A eficiência dos poleiros também é notada quando se compara o número de espécies vegetais que tem seu banco de sementes incrementado ao se utilizar atrativos de pouso para aves. Diversas espécies, como S. mauritianum, C. pachystachya e Psidium guajava L., 1753 (Myrtaceae), apresentaram um número maior de sementes nos coletores dos poleiros distantes da borda da mata, onde não havia concorrência com os poleiros naturais. De acordo com Guedes, Melo e Griffith 
(1997), o posicionamento dos poleiros artificiais em áreas abertas facilita sua visualização pelas aves e os tornam mais efetivos. A distância da mata também foi importante para Mores e Bobrowski (2018), que constataram maior número de sementes em coletores localizados abaixo de poleiros artificiais a $5 \mathrm{~m}$ de distância da floresta. Dias, Umetsu e Breier (2014) observaram que poleiros distribuídos de 5 a $35 \mathrm{~m}$ da borda da floresta foram igualmente frequentados pelas aves e contribuíram com a chuva de sementes nessas áreas, enquanto aqueles posicionados no interior da mata foram menos efetivos.

Entretanto, apesar de os poleiros artificiais contribuírem com a chegada de sementes em áreas degradadas, ainda se discute a capacidade de germinação das sementes nessas localidades, visto que as condições podem não ser favoráveis (TOMAZI; ZIMMERMANN; LAPS, 2010; ALMEIDA et al., 2016). De acordo com Fragoso et al. (2017), poucas pesquisas tratam da importância da preparação do solo sob os poleiros para facilitar a germinação das sementes e o recrutamento de plântulas, sendo uma área de estudo a ser explorada para se estabelecerem melhores parâmetros de usos de poleiros artificiais. No nosso trabalho ficou demonstrado que a utilização de poleiros artificiais aumenta a dispersão de sementes em áreas degradadas ao oferecer locais de pouso para aves. Nesse sentido, trata-se de uma ferramenta barata e efetiva para contribuir em projetos de restauração ambiental.

\section{CONCLUSÃO}

Os poleiros artificiais foram eficazes na atração de aves dispersoras de sementes, principalmente quando distantes $5 \mathrm{~m}$ da borda da mata, fato indicado pelo maior aporte de sementes nos coletores desses poleiros. Trata-se de uma ferramenta eficaz para recomposição de bancos de sementes em áreas degradadas a partir de plantas localizadas em remanescentes florestais próximos, promovendo uma possível recomposição florística semelhante à área de entorno.

\section{REFERÊNCIAS}

ALMEIDA, A. et al. Limited effectiveness of artificial bird perches for the establishment of seedlings and the restoration of Brazil's Atlantic Forest. Journal for Nature Conservation, v. 34, n. 1, p. 24$32,2016$.

ALVARENGA, A. P. Avaliação inicial da recuperação de mata ciliar em nascentes. 2004. 175f. Dissertação (Mestrado em Engenharia Florestal) - Universidade Federal de Lavras, Lavras, 2004.

BARBOSA, J. M. et al. Ecologia da Dispersão de Sementes em Florestas Tropicais. In: S.V. MARTINS, S. V. (Org.). Ecologia de Florestas Tropicais do Brasil. Viçosa: Editora UFV, 2009. p. 52-73.

BARNETT, J. P.; BAKER, J. B. Regeneration Methods. In: DURYEA, M. L.; DOUGHERTY, P. M. (Org.). Forest Regeneration Manual. Dordrecth: Kluver Academic Publishers, 1991. p 35-50.

BOCCHESE, R. A. et al. Chuva de sementes e estabelecimento de plântulas a partir da utilização de árvores isoladas e poleiros artificiais por aves dispersoras de sementes, em área de Cerrado, Mato Grosso do Sul, Brasil. Revista Brasileira de Ornitologia, v. 16, n. 3, p. 207-213, 2008.

BRAGA, F. A.; MENDES, A. O.; FONSECA, A. R. Avaliação de áreas ciliares regeneradas naturalmente às margens do rio itapecerica em Divinópolis, MG. Revista Científica Eletrônica de Engenharia Florestal, v. 15, n. 1, p. 1-12, 2010.

BRASIL. Lei 12.651, de 25 de Maio de 2012. Brasília: Casa Civil, 2012. 
CARPANEZZI, A. A. Fundamentos para a reabilitação de ecossistemas florestais. In: GALVÃO, P. A.; PORFÍRIO-DA-SILVA, V. (Org.) Restauração florestal: fundamentos e estudos de caso. Colombo: EMBRAPA Florestas, 2005. p. 27-45.

CHABARIBERY, D. et al. Recuperação De Matas Ciliares: sistemas de formação de floresta nativa em propriedades familiares. Informações Econômicas, v. 38, n. 6, p. 7-20, 2008.

DIAS, C. R.; UMETSU, F.; BREIER, T. B. Contribuição dos poleiros artificiais na dispersão de sementes e sua aplicação na restauração florestal. Ciência Florestal, v. 24, n. 2, p. 501-507, 2014.

FRAGOSO, R. O. et al. Barreiras ao estabelecimento da regeneração natural em áreas de pastagens abandonadas. Ciência Florestal, v. 27, n. 4, p. 1451-1464, 2017.

GUEDES, M. C.; MELO, V. A.; GRIFFITH, J. J. Uso de poleiros artificiais e ilhas de vegetação por aves dispersoras de sementes. Ararajuba, v. 5. n. 2. p. 229-232, 1997.

GUIDETTI, B. Y. et al. Artificial perches promote vegetation restoration. Plant Ecology, v. 217, n. 7, p. 935-942, 2016.

HAMMER, O.; HARPER, D. A. T.; RYAN, P. D. Past: Paleontological statistics software package for education and data analysis. Palaeontologia Electronica, v. 4, n. 1, p. 9, 2001.

KUNTSCHIK, D. P.; EDUARTE, M.; UEHARA, T. H. K. Matas ciliares. 2. ed. Secretaria do Meio Ambiente, Coordenadoria de Biodiversidade e Recursos Naturais. São Paulo: SMA, 2014. 80 p.

LACERDA, D. M. A.; FIGUEIREDO, P. S. Restauração de matas ciliares do rio Mearim no Município de Barra do Corda-MA: seleção de espécies e comparação de metodologias de reflorestamento. Acta Amazônica, v. 39, n. 2, p.295-304, 2009.

MORES, G. J.; BOBROWSKI, R. Efeitos da presença e distância de poleiros artificiais na dispersão de sementes de uma área degradada em Irati, Paraná. Acta Biológica Catarinense, v. 5, n. 2, p. 106$117,2018$.

REIS, A. et al. Restauração de áreas degradadas: a nucleação como base para incrementar os processos sucessionais. Natureza e Conservação, v. 1, n. 1, p. 28-36, 2003.

RUSCHEL, A. R.; NODARI, R. O. Solanum mauritianum: Fumo-bravo. In: CORADIN, L.; SIMINSKI, A.; REIS, A. (Org.). Espécies Nativas da Flora Brasileira de Valor Econômico Atual ou Potencial. Brasília: Ministério do Meio Ambiente, 2011. p. 692-696.

SAATKAMP, A.; POSCHLOD, P.; VENABLE, D. L. The functional role of soil seed banks in natural communities. In: GALLAGHER R. S. (Org.). Seeds - the ecology of regeneration in plant communities. 3. ed. Wallingford: CABI, 2014. p. 263-295.

SANTOS, R. G.; FRANCHIN, A. G.; NUNES, J. F. Frugivoria por aves em Cecropia pachystachya Trécul (Urticaceae) em uma área verde urbana de passos-MG. Biodiversidade, v. 16, n. 3, p. 16-26, 2017. 
SATO, T. M.; PASSOS, F. C.; NOGUEIRA, A. C. Frugivoria de morcegos (Mammalia, Chiroptera) em Cecropia pachystachya (Urticaceae) e seus efeitos na germinação das sementes. Papéis Avulsos de Zoologia, v. 48, n. 3, p. 19-26, 2008.

SEVEGNANI, L. et al. Flora arbórea e o impacto humano nos fragmentos florestais na bacia do rio Pelotas, Santa Catarina, Brasil. Revista de Estudos Ambientais, v. 14, n. 1, p. 60-73, 2012.

SOUZA, V. C.; LORENZI, H. Botânica sistemática: guia ilustrado para identificação das famílias de fanerógamas nativas e exóticas no Brasil, baseado em APG II. 2. ed. Nova Odessa: Instituto Plantarum, 2008. 704 p.

TOMAZI, A. L.; ZIMMERMANN, C. E.; LAPS, R. R. Poleiros artificiais como modelo de nucleação para restauração de ambientes ciliares: caracterização da chuva de sementes e regeneração natural. Biotemas, v. 23, n. 3, p. 125-135, 2010.

VIBRANS, A. C. et al. Structure of mixed ombrophyllous forests with Araucaria angustifolia (Araucariaceae) under external stress in Southern Brazil. Revista de Biologia Tropical, v. 59, n. 3, p. 1371-1387, 2011. 